\title{
The construction of RFT from the Lipatov's effective action
}

\author{
Sergey Bondarenko ${ }^{1, *}$ and Semyon Pozdnyakov ${ }^{1, * *}$ \\ ${ }^{1}$ Physics Department, Ariel University, Ariel, Israel
}

\begin{abstract}
We consider the formalism of small-x effective action for reggeized gluons, see [1-3]. We construct the perturbation theory based on the knowledge of the classical solutions of equations of motion (written with NNLO precision) and loops contributions to the effective action. Applications of the obtained results are also discussed.
\end{abstract}

\section{Introduction}

The Lipatov effective action, see [2], is a non-linear gauge invariant action which correctly reproduces the production of the particles in direct channels at a quasi-multi-Regge kinematics and written in the covariant form in terms of gluon field $v$ as

$$
S_{e f f}=-\int d^{4} x\left(\frac{1}{4} G_{\mu \nu}^{a} G_{a}^{\mu v}+\operatorname{tr}\left[\left(A_{+}\left(v_{+}\right)-A_{+}\right) j_{r e g}^{+}+\left(A_{-}\left(v_{-}\right)-A_{-}\right) j_{r e g}^{-}\right]\right),
$$

where

$$
A_{ \pm}\left(v_{ \pm}\right)=\frac{1}{g} \partial_{ \pm} O\left(x^{ \pm}, v_{ \pm}\right) ; O\left(x^{ \pm}, v_{ \pm}\right)=P e^{g \int_{-\infty}^{x^{ \pm}} d x^{\prime \pm} v_{ \pm}} .
$$

The Lagrangian of the quark fields

$$
L_{q u a r k}=\bar{\psi}\left(i \gamma^{v} \partial_{v}-m-i g \gamma^{v} T^{a} v_{a v}\right) \psi
$$

will be considered further separately. There are additional kinematical constraints for the reggeon fields $\partial_{-} A_{+}=\partial_{+} A_{-}=0$, Regge kinematics, corresponding to the strong-ordering Sudakov components in the multi-Regge kinematics, see [2] and also [4].

The validity of the effective action approach was confirmed in different calculations, see $[5,6]$.

In the framework with an external source of the color charge introduced, keeping only gluon field depending terms in the Eq. (1) action, we rewrite Eq. (1) as

$$
S_{e f f}=-\int d^{4} x\left(\frac{1}{4} G_{\mu \nu}^{a} G_{a}^{\mu \nu}+v_{-} J^{-}\left(v_{-}\right)+v_{+} J^{+}\left(v_{+}\right)\right),
$$

where

$$
J_{a}^{ \pm}\left(V_{ \pm}\right)=\frac{1}{C(R)} O\left(x^{ \pm}, v_{ \pm}\right) \partial_{i}^{2} A_{a}^{ \pm}
$$

\footnotetext{
*e-mail: sergeyb@ariel.ac.il

**e-mail: semyon.pozdnyakov@gmail.com
} 
where $C(R)$ is the eigenvalue of Casimir operator in the representation $R, C(R)=N$ in the case of adjoint representation.

How we can independently find the form of $J_{a}^{ \pm}\left(v_{ \pm}\right)$currents? We assume for these currents that their variation on gluon fields reproduces the Lipatov's induced currents

$$
\delta\left(v_{ \pm} J^{ \pm}\left(v_{ \pm}\right)\right)=\left(\delta v_{ \pm}\right) j_{\mp}^{i n d}\left(v_{ \pm}\right)=\left(\delta v_{ \pm}\right) j^{ \pm}\left(v_{ \pm}\right),
$$

which posesses a covariant conservation property:

$$
\left(D_{ \pm} j_{\mp}^{\text {ind }}\left(v_{ \pm}\right)\right)^{a}=\left(D_{ \pm} j^{ \pm}\left(v_{ \pm}\right)\right)^{a}=0
$$

and completed $v_{c l \pm 0}=A_{ \pm}$. In fact, it is enough to determine $J_{a}^{ \pm}\left(v_{ \pm}\right)$currents's form.

The paper is organizing as following. In the Section 2 we discuss solutions of the equations of motion, the self-consistency of the equations of motion and it's relations to the transversality condition for a certain combination of the fields. In subsection we solved these equations with the LO accuracy and proved that the solution is the same as that obtained in CGC approach in the limit of zero reggeon field. In the Section 3 we discuss RFT calculus based on the effective action, gluon and quark one loop corrections. The Section 4 is dedicated to extending the formalism of the Lipatov effective action for the case of calculation of production amplitudes in quasi-multi-Regge kinematics at high energy.

\section{The classical equations of motion}

The classical equations of motion for the gluon field $v_{\mu}$ which arise from the action are the following:

$$
\left(D_{\mu} G^{\mu v}\right)_{a}=\partial_{\mu} G_{a}^{\mu \nu}+g f_{a b c} v_{\mu}^{b} G^{c \mu v}=j_{a}^{+} \delta^{v+}+j_{a}^{-} \delta^{\nu-}
$$

The light-cone gauge $v_{-}^{a}=0$ further is applied in the equations of motion and thereafter in the final effective action as well. The topic of this part of report is the search of classical solutions for gluon fields in the form of the expansion in powers of the coupling constant $g$ :

$$
v_{+}^{a}=\sum_{k=0}^{+\infty} g^{k} v_{+k}^{a}\left(A_{+}, A_{-}\right), v^{i a}=\sum_{k=0}^{+\infty} g^{k} v_{k}^{i a}\left(A_{+}, A_{-}\right) .
$$

Indeed, the same Eq. (8) we can rewrite in an another form. Due the covariant derivative presence, we can separate the terms in the equations and rewrite the equations as some recurrence relations between the different terms of the Eq. (9) series. Namely, the fields of $k$ perturbative order will stay in the left-hand side of the equations and the terms which consists with the fields of perturbative order less than $k$ but multiplied on the corresponding order of coupling constant $g$ and denoted as $\bar{j}_{k-1}^{+}, \bar{j}_{k-1}^{-}$and $\bar{j}_{k-1}^{i}$ will stay on the right hand side of equations. Therefore, we obtain:

$$
\begin{gathered}
-\partial_{-}\left[\partial_{i} v_{k}^{i}+\partial_{-} v_{+k}\right]=\bar{j}_{k-1}^{+}, \\
\square v_{k}^{j}-\partial^{j}\left[\partial_{i} v_{k}^{i}+\partial_{-} v_{+k}\right]=\bar{j}_{k-1}^{j}, \\
\square v_{+k}-\partial_{+}\left[\partial_{i} v_{k}^{i}+\partial_{-} v_{+k}\right]=\bar{j}_{k-1}^{-} .
\end{gathered}
$$

Here, among of four equations for three fields, one from the equations provides us with a transversality condition for a certain combination of fields. If this condition is satisfied, then the classical solutions for the fields exist and they have a very simple structure. Taking 
derivatives of the Eq. (10)-Eq. (12) in correspondence to the indexes of the r.h.s of them and summing up the 1.h.s. of the obtained expressions we obtain that the self-consistency of the solutions of the Eq. (10)-Eq. (12) is equivalent to the condition of the transversality being imposed on the r.h.s of the sum:

$$
\partial_{\mu} \bar{j}_{k-1}^{\mu}=0,
$$

that means that the classical solutions of the lower perturbative orders and Eq. (6) induced current substituted in $\bar{j}_{k-1}^{\mu}$ functions must have the form which satisfies the Eq. (13) condition.

Classical solutions for $v_{k}$ when requiring $\partial_{\mu} \bar{j}_{k-1}^{\mu}=0$ are self-consistent and have the form

$$
\begin{aligned}
& v_{k}^{j}=\square^{-1}\left[\bar{j}_{k-1}^{j}-\partial^{j} \partial_{-}^{-1} \bar{j}_{k-1}^{+}\right], \\
& v_{+k}=\square^{-1}\left[\bar{j}_{k-1}^{-}-\partial_{+} \partial_{-}^{-1} \bar{j}_{k-1}^{+}\right] .
\end{aligned}
$$

\subsection{LO equations of motion}

In this part section results LO are compared to the calculations performed in the CGC framework and it is demonstrated that the LO CGC results for the classical field are reproduced in our calculations.

$$
\partial_{i} \partial^{i} v_{a+}=-\partial_{i}^{2} A_{a+}, \quad v_{a+}=A_{a+}
$$

and

$$
\left(D_{+}\left(\partial_{-} v_{i}\right)\right)_{a}=0, \quad v_{i}^{b}=U^{b c}\left(v_{+}\right) \rho_{c i}\left(x^{-}, x_{\perp}\right), \quad D_{+} U\left(v_{+}\right)=0 .
$$

Self-consistency condition, induced current and definition of the $\rho_{c i}\left(x^{-}, x_{\perp}\right)$ function:

$$
-\partial_{i} \partial_{-} v_{a}^{i}=j_{a}^{+}, \quad j_{a}^{+}=-U^{a b}\left(v_{+}\right) \partial_{i} \partial_{-} \rho_{a}^{i}
$$

similarly to CGC approach.

From Lipatov's effective action we have:

$$
\delta\left(v_{+} J^{+}\right)=\frac{1}{N} \delta \operatorname{tr}\left[\left(v_{+x} O_{x} \partial_{i}^{2} A^{+}\right)\right]=-\frac{1}{N} \delta v_{+}^{a} \operatorname{tr}\left[T_{a} O T_{b} O^{T}\right]\left(\partial_{i}^{2} A_{b}^{+}\right)
$$

that in the case of adjoint representation gives:

$$
\delta\left(v_{+} J^{+}\right)=\frac{1}{N}\left(\delta v_{+}^{a}\right) \operatorname{tr}\left[f_{a} O f_{b} O^{T}\right]\left(\partial_{i}^{2} A_{b}^{+}\right)=\frac{1}{N}\left(\delta v_{+}^{a}\right) U^{a b}\left(\partial_{i}^{2} A_{b}^{+}\right)
$$

that provides

$$
U^{a b}=\operatorname{tr}\left[f_{a} O f_{b} O^{T}\right]
$$

and

$$
\partial_{i} \partial_{-} \rho_{a}^{i}=-\frac{1}{N} \partial_{\perp}^{2} A_{a}^{+}, \quad \rho_{a}^{i}=\frac{1}{N} \partial_{-}^{-1}\left(\partial^{i} A_{-}^{a}\right),
$$

connection with CGC approach. 


\section{LO RFT}

Inserting obtained classical gluon fields solutions $v_{c l}$ in the Eq. (4) $S_{e f f}$ action, we will obtain an action which will depend only on the reggeon fields, determining the LO RFT action of the approach. Formally, due to the presence of the path-ordered exponential in the solutions, the action will include all orders of perturbative terms which can be important for large $v_{+} \approx A_{+}$in the processes where some large color charge is created. The expansion of these exponential must be supplemented by solution of equations of motion to corresponding orders, otherwise only part of the usual perturbative corrections will be accounted.

In general, the following expansion for the action exists:

$$
S_{e f f}=-\int d^{4} x\left(s_{1}\left[g, A_{+}, A_{-}\right]+g s_{2}\left[g, A_{+}, A_{-}\right]+\cdots\right) .
$$

So $S_{e f f}$ can be expanded in terms of reggeon fields $A_{-}$and $A_{+}$as

$$
\Gamma=\sum_{n, m=0}\left(A_{+}^{a_{1}} \cdots A_{+}^{a_{n}} K_{b_{1} \cdots b_{m}}^{a_{1} \cdots a_{n}} A_{-}^{b_{1}} \cdots A_{-}^{b_{m}}\right),
$$

that determines this expression as functional of reggeon fields and provides effective vertices of the interactions of the reggeized gluons in the RFT calculus.

Substituting the LO of classical solutions into action gives us the full LO value of the kernel only for $n+m=2$ and only a fraction of the contributions for more complex vertices. NLO of classical solutions allows us to get a complete answer for $n+m=3$. With NNLO of classical solutions, we moved on to 4-reggeon interactions.

\subsection{One loop expression}

We define:

$$
v_{i}^{a} \rightarrow v_{i c l}^{a}+\varepsilon_{i}^{a}, \quad v_{+}^{a} \rightarrow v_{+c l}^{a}+\varepsilon_{+}^{a}
$$

and expand the light-cone Lagrangian around the non-trivial classical solutions. We need to consider only contributions of no higher than second order in the fluctuations $\varepsilon^{a}$ for a oneloop correction. We integrate the expression for these fluctuations $\varepsilon^{a}$. Final expression for the one loop effective action:

$$
\begin{aligned}
\Gamma & =\int d^{4} x\left(L_{Y M}\left(v_{i}^{c l}, v_{+}^{c l}\right)-v_{+c l}^{a} J_{a}^{+}\left(v_{+}^{c l}\right)-A_{+}^{a}\left(\partial_{i}^{2} A_{-}^{a}\right)\right) \\
& +\frac{l}{2} \ln \left(1+G\left(v^{c l}\right) M\left(v^{c l}\right)\right),
\end{aligned}
$$

which is functional of the reggeized gluon fields only. These actions and equation (26) are published in detail in [7].

The interaction of reggeized gluons $A_{+}$and $A_{-}$is defined as effective vertex of interactions of reggeon fields in the action:

$$
\left(K_{x y}^{a b}\right)^{+-}=K_{x y}^{a b}=\left(\frac{\delta^{2} \Gamma}{\delta A_{+x}^{a} \delta A_{-y}^{b}}\right)_{A_{+}, A_{-}=0},
$$

we can call this vertex as interaction kernel as well.

There are also other kernels, related to $\left\langle A_{+} A_{+}\right\rangle$and $\left\langle A_{-} A_{-}\right\rangle$propagators, in the leading order the contributions to these kernels are zero: 


$$
\left(K_{x y}^{a b}\right)_{0}^{++}=\left(K_{x y}^{a b}\right)_{0}^{--}=0 .
$$

We represent the interaction kernels $A_{+}, A_{-}$in the form of an expansion in powers of $g^{2}$

$$
K_{z w}^{b d}=\sum_{k=0}^{\infty} K_{z w k}^{b d} .
$$

The contributions to this kernel are provided by the different terms in the action which are linear with respect to $A_{+}, A_{-}$fields. For example, the variation of a logarithms gives:

$$
\begin{aligned}
-2 \imath K_{x y}^{a b} & =\left(\frac{\delta^{2} \ln (1+G M)}{\delta A_{+x}^{a} \delta A_{-y}^{b}}\right)_{A_{+}, A_{-}=0} \\
& =\left[\left(\frac{\delta^{2} G}{\delta A_{+x}^{a} \delta A_{-y}^{b}} M+\frac{\delta G}{\delta A_{+x}^{a}} \frac{\delta M}{\delta A_{-y}^{b}}+\frac{\delta G}{\delta A_{-y}^{b}} \frac{\delta M}{\delta A_{+x}^{a}}+G \frac{\delta^{2} M}{\delta A_{+x}^{a} \delta A_{-y}^{b}}\right)(1+G M)^{-1}\right. \\
& \left.-\left(\frac{\delta G}{\delta A_{-y}^{b}} M+G \frac{\delta M}{\delta A_{-y}^{b}}\right)(1+G M)^{-1}\left(\frac{\delta G}{\delta A_{+x}^{a}} M+G \frac{\delta M}{\delta A_{+x}^{a}}\right)(1+G M)^{-1}\right]_{A_{+}, A_{-}=0} .
\end{aligned}
$$

Leading order contribution:

$$
K_{x y 0}^{a b}=-\delta^{a b} \delta_{x y} \partial_{i x}^{2}=\delta^{a b} \delta_{x y}\left(\partial_{i} \partial^{i}\right)_{x} .
$$

For the kernel $K_{z w}^{b d}=\sum_{k=0} K_{z w k}^{b d}$ the propagator is defined as

$$
\int d^{4} z\left(K_{x z}^{a b}\right)^{-+}\left(D_{z y}^{b c}\right)_{+-}=\delta^{a c} \delta_{x y}
$$

or

$$
\begin{aligned}
\left(D_{x y}^{a c}\right)_{+-} & =\left(D_{x y}^{a c}\right)_{0+-}-\int d^{4} z \int d^{4} w\left(D_{x z}^{a b}\right)_{0+-}\left(\left(K_{z w}^{b d}\right)^{-+}\right. \\
& \left.-\left(K_{z w}^{b d}\right)_{0}^{-+}\right)\left(D_{w y}^{d c}\right)_{+-},
\end{aligned}
$$

where

$$
\int d^{4} z K_{x z 0}^{a b} D_{z y 0}^{b c}=\delta^{a c} \delta_{x y} .
$$

The well-known NLO kernel:

$$
-2{ }_{\imath} K_{x y 1}^{a b}=\frac{\imath g^{2} N}{4 \pi} \partial_{i x}^{2}\left(\int \frac{d p_{-}}{p_{-}} \int \frac{d^{2} p_{\perp}}{(2 \pi)^{2}} \int \frac{d^{2} k_{\perp}}{(2 \pi)^{2}} \frac{k_{\perp}^{2}}{p_{\perp}^{2}\left(p_{\perp}-k_{\perp}\right)^{2}} e^{-\imath k_{i}\left(x_{i}-y_{i}\right)}\right)
$$

provides the reggeized gluon propagator equation after the Fourier transform:

$$
\tilde{D}^{a b}\left(p_{\perp}, p_{-}\right)=\frac{\delta^{a b}}{p_{\perp}^{2}}-\frac{g^{2} N}{32 \pi^{3}} \int \frac{d k_{-}^{\prime}}{k_{-}^{\prime}} \int d^{2} k_{\perp} \frac{p_{\perp}^{2}}{k_{\perp}^{2}\left(p_{\perp}-k_{\perp}\right)^{2}} \tilde{D}^{a b}\left(p_{\perp}, k_{-}^{\prime}\right),
$$

here we used

$$
\begin{gathered}
D_{0}^{a b}(x, y)=\delta^{a b} \int \frac{d^{4} p}{(2 \pi)^{4}} \frac{e^{-l p(x-y)}}{p_{\perp}^{2}}, \\
\epsilon\left(p_{\perp}^{2}\right)=-\frac{\alpha_{s} N}{4 \pi^{2}} \int d^{2} k_{\perp} \frac{p_{\perp}^{2}}{k_{\perp}^{2}\left(p_{\perp}-k_{\perp}\right)^{2}} .
\end{gathered}
$$


Rewriting this equation in the differential form:

$$
\frac{\partial \tilde{D}^{a b}\left(p_{\perp}, \eta\right)}{\partial \eta}=\tilde{D}^{a b}\left(p_{\perp}, \eta\right) \epsilon\left(p_{\perp}^{2}\right) .
$$

On the rapidity interval of interest $0<\eta<Y=\ln \left(s / s_{0}\right)$ we obtain the final expression for the propagator:

$$
\tilde{D}^{a b}\left(p_{\perp}, Y\right)=\frac{\delta^{a b}}{p_{\perp}^{2}}\left(\frac{s}{s_{0}}\right)^{\epsilon\left(p_{\perp}^{2}\right)}
$$

\subsection{One loop quark corrections}

The full NNLO contribution to the classical solutions of the gluon equations of motion must include the contribution from the fermion loop as well, see Eq. (4). Expanding this part of the Lagrangian around $\psi=\bar{\psi}=0^{1}$ classical solutions we find only one correction of the order $g^{2}$ and $\varepsilon^{2}$. This addition will allow us to take into account the contribution with a quark loop to the gluon fields. Integrating with respect the fluctuation we obtain the following expression for the contribution to the action:

$$
-\frac{g^{2}}{4} \operatorname{tr}\left(G_{q}^{0}(y, x)\left(\gamma^{v} v_{a v}(x)\right) G_{q}^{0}(x, y)\left(\gamma^{\mu} v_{\mu}^{a}(y)\right)\right)
$$

where

$$
G_{q}^{0}(x, y)=\left(i \gamma_{v} \partial_{x}^{v}+m\right) \Delta_{x y}, \quad \Delta_{x y}=\int \frac{d^{4} p}{(2 \pi)^{4}} \frac{e^{i p(x-y)}}{p^{2}-m^{2}+i 0} .
$$

Then after varying by $v_{a \rho}(z)$ we obtain the following contributions to the equation of motion

$$
\left.j_{\text {quark } a}^{\rho}(z)=-\frac{g^{2}}{2} \operatorname{tr}\left(\gamma^{\rho} G_{q}^{0}(z, y) \gamma^{\mu} v_{a \mu}(y) G_{q}^{0}(y, z)\right)\right) .
$$

We verified, that condition $\partial_{\rho} j_{\text {quark } a}^{\rho}=0$ is true. Then the classical solutions have additional contributions $v_{2 a}^{i}$ and $v_{+2 a}$, respectively:

$$
\begin{aligned}
v_{q 2 a}^{i} & =\square^{-1}\left[j_{\text {quark } 0 a}^{i}-\partial^{i} \partial_{-}^{-1} j_{\text {quark } 0 a}^{+}\right], \\
v_{q+2 a} & =\square^{-1}\left[j_{\text {quark } 0 a}^{-}-\partial_{+} \partial_{-}^{-1} j_{\text {quark } 0 a}^{+}\right] .
\end{aligned}
$$

For example, we estimated the contribution of quarks to the reggeon propagator. We have two additional contributions to the effective action from the inclusion of quarks: direct contribution to the effective action and contribution from expansion of the classical solution $v_{a}^{i}$ and $v_{+a}$ by (44) and (45), respectively. After the calculations, we showed that this contributions are zero. That means that the reggeon propagator does not change from taking the quark loop contribution into account, that is well known result, see [8].

\footnotetext{
${ }^{1}$ In the approach we separate the classical solutions for the quark fields and fluctuations around these classical solution, in general the non-zero classical quark fields correspond to the production amplitudes in the approach.
} 


\section{The description of gluon and quark production amplitudes}

We extend the formalism in order to incorporate the vertices of real particles production, i.e. the vertices of reggeons interaction with asymptotic gluon and quark fields.

In order to compute the production amplitudes in the quasi-multi-Regge kinematics, the classical solutions must be modified taking into account solutions of equations of motion for free gluon field $v_{f}^{\mu}$ :

$$
v_{c l}^{\mu} \rightarrow v_{c l}^{\mu}+v_{f}^{\mu} .
$$

The $v_{c l}^{\mu}$ fields were calculated in our work to NNLO precision, here we consider the equations of motion for $v_{f}^{\mu}$

$$
\left(D_{\mu} G^{\mu v}\right)_{a}=0
$$

to $\mathrm{LO}$ precision.

These equations provide the well-known expressions for $v_{+}$and $v_{\perp}$.

The first equation provides a constraint of the theory in light-cone gauge:

$$
v_{a+f}=-\partial_{-}^{-1} \partial^{i} v_{a i f} .
$$

The second equation of motion for the dynamical $v_{i}$ fields:

$$
\square v_{\text {aif }}=0 .
$$

The third equation can be considered as a check of consistency of the found solutions, it is equal to zero by construction. We see also, that the Lorentz condition for the free gluon field

$$
\partial^{\mu} v_{a \mu f}=0
$$

is satisfied in this case as an operator equation.

Considering the fermionic part of the QCD Lagrangian, we follow the usual light-cone decomposition of the quark field. Introducing projector operators

$$
\Lambda^{ \pm}=\frac{\gamma^{\mp} \gamma^{ \pm}}{2}
$$

we project out the fermion field obtaining two two-component spinors:

$$
\psi_{ \pm}=\Lambda^{ \pm} \psi
$$

The free, asymptotic quark fields can be defined as solutions of equations of motion obtained from the free quarks Lagrangian

$$
L_{Q}^{f}=i \sqrt{2} \psi_{+}^{\dagger} \partial_{+} \psi_{+}-\frac{i}{\sqrt{2}} \psi_{+}^{\dagger}\left(i \gamma^{\perp} \partial_{\perp}+m\right) \partial_{-}^{-1}\left(i \gamma^{\perp} \partial_{\perp}-m\right) \psi_{+}
$$

and which reads as

$$
\left(\square+m^{2}\right) \psi_{+f}=0 .
$$

With the new classical solution the effective action becomes a functional of the Reggeon, free gluon and quark fields: $\Gamma\left(A_{+}, A_{-}, v_{f}, \psi_{+f}^{\dagger}, \psi_{+}\right)$. Now, the QCD S-matrix generating functional of the theory consists with free gluon and quark fields we can write as

$$
S\left(v_{f}, \psi_{+f}^{\dagger}, \psi_{+f}\right)=\int d A e^{i \Gamma\left(A_{+}, A_{-}, v_{f}, \psi_{+f}^{\dagger}, \psi_{+f}\right)}
$$


We can calculate any vertex of the theory which consists with the fields, vertex of interaction of $n A_{+}+m A_{-}+k v_{\perp f}+p \psi_{+f}+p \psi_{+f}^{\dagger}$ fields that could be written as:

$$
\left(K_{a_{1} \cdots a_{n+m}}\right)_{p ; p}^{c_{1} \cdots c_{k}}=\left(\frac{\delta^{n+m+k+2 p} \Gamma\left(A_{+}, A_{-}, v_{f}, \psi_{+f}^{\dagger}, \psi_{+f}\right)}{\delta A_{ \pm}^{a_{1}} \cdots \delta A_{ \pm}^{a_{n+m}} \delta v_{\perp f}^{c_{1}} \cdots \delta v_{\perp f}^{c_{k}} \delta \psi_{+f}^{1} \cdots \delta \psi_{+f}^{p} \delta \psi_{+f}^{1 \dagger} \cdots \delta \psi_{+f}^{p^{\dagger}}}\right),
$$

where as usual we take all the fields equal to zero after the derivatives taken.

\section{Conclusion}

This proceeding provides an overview of the construction of RFT from the Lipatov's effective action. This topic is discussed in more detail in articles [2, 7, 9-11]. The action (55) and (26), which depends only on reggeons and asymptotically free gluons and quarks, is derived. Any vertex of the theory which consists with these fields can be obtained from this action Eq. (56) with the accuracy of one-loop corrections for gluons and quarks contributions. The way how to extend this action to a greater number of loops is shown. The closest problem that is to verify our results is the calculation of the vertices of Lipatov and Fadin with the accuracy of one-loop corrections. In paper [12], we derived the Dayson-Schwinger hierarchy of the equations for the correlators of the reggeized gluon fields in the framework of this formalism. This is an extension of work [4], which discussed the correspondence between the correlators of reggeized gluon fields and Wilson line operators of longitudinal gluon field.

\section{References}

[1] V. N. Gribov, Sov. Phys. JETP 26, 414 (1968).

[2] L. N. Lipatov, Nucl. Phys. B 452, 369 (1995); Phys. Rept. 286, 131 (1997); Subnucl. Ser. 49, 131 (2013); Int. J. Mod. Phys. Conf. Ser. 39, 1560082 (2015); Int. J. Mod. Phys. A 31, no. 28/29, 1645011 (2016); EPJ Web Conf. 125, 01010 (2016).

[3] L. N. Lipatov, Sov. J. Nucl. Phys. 23, 338 (1976) [Yad. Fiz. 23 (1976) 642]; E. A. Kuraev, L. N. Lipatov and V. S. Fadin, Sov. Phys. JETP 45, 199 (1977) [Zh. Eksp. Teor. Fiz. 72, 377 (1977)]; I. I. Balitsky and L. N. Lipatov, Sov. J. Nucl. Phys. 28, 822 (1978) [Yad. Fiz. 28, 1597 (1978)].

[4] S. Bondarenko and M. A. Zubkov, Eur. Phys. J. C 78 no.8, 617 (2018).

[5] L. N. Lipatov, Nucl. Phys. Proc. Suppl. 99A, 175 (2001); M. A. Braun and M. I. Vyazovsky, Eur. Phys. J. C 72, 1864 (2012); M. Hentschinski and A. Sabio Vera, Phys. Rev. D 85, 056006 (2012); M. A. Braun, M. Y. Salykin, S. S. Pozdnyakov and M. I. Vyazovsky, Eur. Phys. J. C 72, 2223 (2012); J. Bartels, L. N. Lipatov and G. P. Vacca, Eur. Phys. J. C 77 no.7, 498 (2017); M. Hentschinski, arXiv:1802.06755 [hep-ph].

[6] M. Nefedov, V. Saleev, Mod. Phys. Lett. A 32 no.40, 1750207 (2017).

[7] S. Bondarenko, L. Lipatov, S. Pozdnyakov, A. Prygarin, Eur. Phys. J. C 77 no.9, 630 (2017).

[8] V. S. Fadin and R. Fiore, Phys. Lett. B 294, 286 (1992); V. S. Fadin, R. Fiore and A. Quartarolo, Phys. Rev. D 50, 2265 (1994); V. S. Fadin, R. Fiore and A. Quartarolo, Phys. Rev. D 50, 5893 (1994); V. S. Fadin, R. Fiore, A. Flachi and M. I. Kotsky, Phys. Lett. B 422, 287 (1998).

[9] S. Bondarenko, L. Lipatov and A. Prygarin, Eur. Phys. J. C 77 no.8, 527 (2017).

[10] S. Bondarenko, S. Pozdnyakov, arXiv:1802.05508 [hep-ph].

[11] S. Bondarenko, S. Pozdnyakov, Phys. Lett. B 783, 207 (2018)

[12] S. Bondarenko, S. Pozdnyakov, arXiv:1806.02563 [hep-th]. 